\author{
In-Space Cryogenic Propellant Depot Stepping Stone \\ IAC-05_D3.2.01 \\ $56^{\text {th }}$ International Astronautical Congress \\ October 17-21, 2005 \\ Fukuoka, Japan \\ Joe T. Howell \\ NASA Marshall Space Flight Center \\ Advanced Projects Team, SP20 \\ Huntsville, AL 35812 USA \\ E-mail: Joe.Howell@,nasa.gov \\ John C. Mankins \\ Artemis Innovation Management Solutions, LLC \\ Ashburn, VA 20147 USA \\ E-mail: john.c.mankins@,artemisinnovation.com \\ John C. Fikes \\ Advanced Projects Team \\ NASA Marshall Space Flight Center \\ Huntsville, AL 35812 USA \\ E-mail: john.c.fikes@nasa.gov
}

\begin{abstract}
$\underline{\text { Abstract }}$
An In-Space Cryogenic Propellant Depot (ISCPD) is an important stepping stone to provide the capability to preposition, store, manufacture, and later use the propellants for Earth-Neighborhood campaigns and beyond. An in-space propellant depot will provide affordable propellants and other similar consumables to support the development of sustainable and affordable exploration strategies as well as commercial space activities. An in-space propellant depot not only requires technology development in key areas such as zero boil-off storage and fluid transfer, but in other areas such as lightweight structures, highly reliable connectors, and autonomous operations. These technologies can be applicable to a broad range of propellant depot concepts or specific to a certain design. In addition, these technologies are required for spacecraft and orbit transfer vehicle propulsion and power systems, and space life support. Generally, applications of this technology require long-term storage, on-orbit fluid transfer and supply, cryogenic propellant production from water, unique instrumentation and autonomous operations.

This paper discusses the reasons why such advances are important to future affordable and sustainable operations in space. This paper also discusses briefly $R \& D$ objectives comprising a promising approach to the systems planning and evolution into a meaningful stepping stone design, development, and implementation of an In-Space Cryogenic Propellant Depot. The success of a well-planned and orchestrated approach holds great promise for achieving innovation and revolutionary technology development for supporting future exploration and development of space.
\end{abstract}




\section{The Sustainability Challenge}

One of the central challenges to more ambitious - yet still affordable - space operations in the Earth's neighborhood lies in our inability to affordably preposition consumables and needed systems (including spares for in space servicing and maintenance). This requirement is especially critical in the case of propellants -including propellants for vehicles transporting crew or time-critical payloads. As long as it is not possible to locally repair and refuel high-value (and high-cost) space systems beyond low Earth orbit (LEO). This challenge affects planning for a wide range of potential future missions, but is particularly important for (a) major, highvalue missions such as human exploration activities beyond low Earth orbit; (b) largescale defense and/or security focused mission systems; or, (c) 'future space industries' (such as larger, multi-payload geostationary Earth orbit (GEO) platforms, space solar power systems, and related concepts).

For example, a large-scale, permanently inhabited lunar base might involve 3-4 human missions to the Moon per year (for crew rotation every 120 days or 90 days, respectively). However, if such a mission scenario were to involve Apollo-era concepts and current-technology expendable space transportation systems, then the total cost per mission due to transportation costs alone (hardware and operations), could range from $\$ 2,400 \mathrm{M}$ per mission to more than $\$ 3,100 \mathrm{M}$ per mission (current year dollars). Transportation cost components here are assumed to include the following:

- ETO Transport: Assuming Shuttle-derived, expendable systems involving 2 Heavy Lift Launch Vehicles (HLLVs), 1 Crew Launcher at a total cost of $\$ 500 \mathrm{M}$ to $\$ 1,000 \mathrm{M}$ per mission. Note that this rough estimate for ETO costs is intended to be comparable to (but lower than) the Space Shuttle at about 3-4 launches per year, plus typical EELV (evolved expendable launch vehicles) costs per launch at the same rate.

- In Space Transport: Assuming expendable systems involving at least two in-space stages with individual mass of about 10,000 $\mathrm{kg}$ (and a recurring unit per kilogram cost of about $\$ 50,000$ per kilogram ${ }^{1}$ ) for a "per mission cost" of about $\$ 500 \mathrm{M}$ per mission.

- Excursion Transport: Assuming expendable systems, involving nominally a descent module and an ascent module with a combined mass of about $10,000 \mathrm{~kg}$ to $20,000 \mathrm{~kg}$ (and a recurring unit per kilogram cost of approximately $\$ 50,000$ per kilogram), for a "per mission cost" of about $\$ 750 \mathrm{M}$ per mission.

- Transportation Operations. Assuming incremental improvements on Space Shuttle and International Space Station (ISS) era ground operations concepts, involving nominally about 20,000 total personnel (with an average cost of about $\$ 100,000$ per FTE (full time equivalent) $)^{2}$, for a "program per year cost" of about $\$ 2,000 \mathrm{M}$, and a per mission cost of about $\$ 500 \mathrm{M}$ per mission at a rate of 4 missions per year, or about to $\$ 667 \mathrm{M}$ per mission at a rate of 3 missions per year.

\footnotetext{
${ }^{1}$ The very rough, mass-based cost estimation relationships (CERs) used in this illustration are intended to be consistent with - and perhaps a bit on the optimistic side of - past human-rated space systems recurring hardware costs. A more detailed analysis would consider the specific cost per kilogram for each of the major elements in each system, as well as taking into account the specific number of unique elements to be manufactured over the life of the program, and the degree to which advanced production methods (such lean manufacturing) might be brought to bear on the problem. However, for the purposes of this discussion, the single value of 'about $\$ 50,000$ per kilogram (about $\$ 22,000$ per pound) for recurring flight hardware costs seems adequate.

${ }^{2}$ This figure is intended to be a very rough average, integrating all personnel involved; clearly some cost categories, such as senior engineers, are at much higher labor rates when 'fully wrapped'.
} 
In summary, this scenario would result in an annual cost-for lunar base transportation only — of approximately $\$ 7,000 \mathrm{M} /$ year (best case, 3 missions/year), to about $\$ 11,000 \mathrm{M} /$ year (worst case, 4 missions per year). Additional costs would, of course be incurred for crew transportation systems, supporting infrastructures (such as communications systems), as well as for the wide range of surface systems that would be needed for a lunar base. (It is perhaps worth noting that in the case of the tightly interwoven Space Shuttle and ISS programs, the costs of transportation to and from the Station are very roughly equivalent to the costs of ISS engineering and operations. If the same were to hold true for a far-more technically challenging lunar base and its transportation system, then the total annual costs would be double the figures noted above - or equivalent something greater than the entire current U.S. annual civil space budget.) The total of such annual operational costs would, of course, far exceed the current annual US investment in human space flight. Moreover, they would not allow for vitally needed investments in the systems that would allow us to go beyond and initial operational base.

Although the sketch above is specific to a notional lunar base, the affordability issues involved are quite similar for a range of other ambitious future space operational scenarios - particularly those involving (as does the Terrestrial Planet Imager (TPI) concept) a number of exceptionally large, high-value imaging systems deployed beyond low Earth orbit (LEO).

\section{A Key Element of a Notional Solution}

One potential solution to this challenge is to move successfully to more affordable reusable space transportation system elements with substantially higher levels of onboard autonomy. Four functional challenges must be resolved to enable this highly desirable, but technical difficult transition:

- Lower cost ETO transport (perhaps by enabling a transition to launchers that are more similar to those used by other government organizations or by commercial sectors; and in the long term by transitioning to reusable launch vehicles);

- Highly-autonomous assembly, maintenance and servicing of modular systems in space and on planetary surfaces (including both robotic and crew-assisted operations),

- Affordable and timely pre-positioning of fuel, systems and other materiel throughout the Earth-Moon system (including to the surface of the Moon); and,

- Reusable, highly reliable and high-energy in space transportation (and for lunar missions, excursion transportation systems).

The systems that would enable such visionary capabilities must also be highly autonomous (to reduce ground operations costs), as well as substantially less expensive to buy and own (with greater operational margins than current systems, as well as lower per unit costs - perhaps achieved through modularity and the economies of production).

Detailed studies would be needed to determine the appropriate technical performance objectives for such advanced systems - in the context of cost constraints and reliability (safety) goals. However, it seems plausible to suggest that at a minimum, such future R\&D efforts should target new systems approaches and novel technologies that would make possible not less that a factor of four reduction in per mission costs, and perhaps as much as a 10fold reduction. In the case of the lunar base example sketched above, a 4-fold reduction 
would be equivalent to seeking to achieve a lunar base per mission transportation cost of no more than $\$ 1,750 \mathrm{M}$ to $\$ 2,750 \mathrm{M}$ per mission - or, in the case of a 10-fold reduction, a per mission transportation cost of no more than $\$ 700 \mathrm{M}$ to $\$ 1,100 \mathrm{M}$ per mission. (For comparison, note that the latter figures are roughly comparable to the fully-loaded costs of Space Shuttle missions to LEO at the present time-although they are still much greater than the marginal costs of such flights.)

However, setting a goal is hardly the same as achieving it. Although the technologies needed to achieve this vision are (in many cases) already validated in the laboratory, they are certainly not 'in hand' or sufficiently mature to incorporate into space systems being build today. As a result, substantial research and technology development and validation must still be undertaken in order to realize the potential cost savings that are so clearly needed in end-to-end space transportation.

Fortunately, the capabilities for local refueling, as well as locally autonomous assembly, repair and maintenance are inherent for any kind of extended and ambitious deep space scenario-such as a lunar surface base. ${ }^{3}$ Moreover, they become even more critical as one considers the longterm requirements of human mission to Mars, much less the far more ambitious requirements of extended human presence and activity in space (e.g., space settlements or missions beyond the inner solar system).

\footnotetext{
${ }^{3}$ Note: although smaller missions-such as those involving traditional communications satellitesmight benefit from in-space refueling, low-cost in space transportation and similar space operations, the costs of developing and deploying such transformational new capabilities are very difficult, if not impossible to justify based on these missions alone.
}

As a result, the future development of such technologies should be broadly beneficial to the full range of ambitious mission options that are under consideration by various organizations.

\section{$\underline{\text { Key Technical Challenges }}$}

The central functional issues associated with affordably realizing advanced, highly reusable architectural concepts lies in solving several key technology challenges. These include:

- Tele-supervised (and eventually autonomous) highly-resilient deep space systems operations (in this case, 'deep space' operations includes all ambitious mission operations beyond LEO).

- Reconfigurable and self-adaptive modular systems.

- Space assembly, maintenance and servicing (from the systems level, down to the subsystem level).

- Highly fuel-efficient, high reliability, restartable propulsion, such as high-power electric propulsion for cargo and cryogenic engines for time critical mission (such as those involving astronaut crews).

- High-energy propellants for longduration missions (particularly cryogenic propellants such as liquid oxygen, liquid hydrogen, etc.)

- High-power, but low-mass space power generation and management systems

- Long-term storage and management, as well as the highly reliable and low-loss transfer (including transfer in microgravity) of cryogenic propellants.

This paper will deal with a specific class of these technology problems: those that involve the long-term storage and 
management, and transfer of cryogenic propellants in low gravity.

\section{A New Capability: ISCPD}

In-Space Cryogenic Propellant Depot (ISCPD) systems represent a broad class of new concept of operations with the potential to meet several of the important challenge of enabling affordable pre-positioning of key logistics (including fuel, hardware, and appropriate systems) to points beyond LEO.

The basic concept of an ISCPD system may be pursued in a variety of alternate system configurations - given that the key technology areas have been addressed successfully. Figures 1 and 2 illustrate two notional approaches to cryogenic propellant depots; both involving large, in-space assembled structures and a depot that remains in a low Earth orbit (LEO).

Another rather different approach is that of the "hybrid propellant module" (HPM) in which the fuel depot itself is modular and the individual modules are small enough to be transported individually using a system such as a solar electric propulsion (SEP) orbital transfer vehicle (OTV). Figure 3 illustrates the HPM concept. However, the HPM may also be integrated with others, or with a 'stack' of vehicle elements to form and operational vehicle. Figure 4 illustrates this conceptual approach - with the cryogenic propellant depot element (the HPM) integrated with other elements to form an operational vehicle that may in turn leave LEO with high value, time-critical cargos.

For any of these options, cryogenic propellants will at first require transport from Earth to LEO for subsequent transfer, storage and later use in LEO or elsewhere. Figure 5 illustrates one such concept for a 25-ton class cryogenic propellant tank. (In this concept, the tank is transported to LEO on an Evolved Expendable Launch Vehicle (EELV) and is itself expendable.

In either case, the concept provides for payload attachments on each module to provide flexibility and re-configurability options for accommodating multiple technology experiments and eventually different exploration payloads. In the case of a linear configuration, the platform may also provide a single, larger payload attachment at the central of gravity of the integrated vehicle.

At present, there are still unresolved research issues involved in two-phase cryogenic fluids management. Moreover, ISCPD systems entail considerably greater system development uncertainty than more conventional systems and technologies (e.g., fully expendable, Apollo-era concepts with technologies that are already at TRL 7-9). As a result, significant research and technology is needed prior to beginning major systems development for architectures that involve such concepts. However, if affordability and sustainability are important characteristics for future transformational space operations, then the development of in space refueling is essential.

\section{Needed ISCPD Research and}

\section{Development}

A number of important technology areas must be advanced to enable ISCPD capabilities for future space operations. First, an in-space depot will be required to continuously supply vapor-free cryogenic liquids to an orbital transfer vehicle at an acceptable flow rate and pressure drop. Also to meet a depot efficiency metric of less than $2 \%$ fluid residuals, a highly-capability cryogenic liquid acquisition device (LAD) 
must be designed and developed. R\&D is needed to characterize and develop a design database for fundamental screen wicking characteristics, surface tension data, and screen channel outflow performance with cryogenic fluids.

In addition, low gravity mass gauging with an accuracy of better than 5\% of fill tank will be required for the in space depot. Currently no mass gauge at the desired accuracy level exists for low gravity application. Serveral different approaches to low gravity mass gauging have been identified. Two promising concepts are the Compression Mass Gauge (CMG) and the Optical Mass Gauge (OMG). For both of these, reference mass gauging accuracy data is needed, and previous functional and performance data must be documented. Following further R\&D - in combination with analyses to better define depot architecture derived operational requirements-it will be possible to downselect to a single mass gauge concept to be matured further.

Minimal or zero loss of cryogenic propellants (i.e., "zero boil-off”, or "ZBO") during storage for long durations on an inspace depot is critical for any exploration mission success. Boil-off losses exceeding $3 \%$ per month require excess propellant storage margins and translate into significantly large Earth-to-orbit launch capability. Efforts are needed to develop an efficient, low mass depot concept through upgrades of existing thermal analysis tools. Further development is also needed of a Cryogenic Analysis Tool (CAT) that can quantify system weight comparisons between passive insulation systems and $\mathrm{ZBO}$ systems such as cryo-coolers and radiators for a defined scenario-including specified cryogenic fluid, environment, and quantity). (A partially-validated CAT was previously developed by NASA's Glenn Research Center (GRC) and Ames Research (ARC)). In addition, continuing R\&D to develop new, more efficient and longerlived cryo-coolers is also important. In the area of ZBO and other topics, flight-like components must be developed for use in future systems-level technology validation testing.

Present in-space depot architectures require liquid oxygen (LOX) as an oxidizer for different propellant options. The use of LOX presents various fluid management challenges, with must be resolved through both component level research as well reduced scale depot systems-level testing with LOX. The objectives of such testing would be to validate system designs, provide LOX ZBO and associated pressure control data, validate scaling parameters for active thermal management, and investigate the LOX properties effects on propellant management.

Another in-space depot operational requirement is to assure the "leak-free" transfer of cryogenic propellants during mating and de-mating operations. Efforts are needed to develop and test secondgeneration miniaturized leak sensors for broad application use in propellant depots, in-space transportation vehicles, and launch vehicles.

Nearer-term R\&D must establish a basis for subsequent technology flight demonstrations (TFDs) of cryogenic fluid management (CFM) technologies requiring a low-g environment, ultimately leading to an operational system supporting a human and robotics space infrastructure. To accomplish this goal, architecture and cost/benefit trade studies are needed, in conjunction with test article and facility upgrades, analytical tool development and verification, and leverage 
of existing hardware, facilities, and on-going tests. It is important to build on the synergism to be achieved through a collaboration of experts in the field of cryogenic fluids physics and also to develop a new generation of experienced and skilled personnel to support this critical long-term exploration capability. The goal of such efforts would be to assess and mature the technologies that support future development of modular architectures and systems involving regular, low-cost in space refueling.

\section{$\underline{\text { Summary and Conclusions }}$}

Without substantial systems-level innovation and the development of tractable, but as yet un-demonstrated new technologies, a broad range of ambitious space operations beyond low Earth orbit cannot become either affordable or sustainable. The servicing of propellants and other consumables in space enables a many mission scenarios, otherwise unavailable due to costs, operational constraints, or inefficiencies.

Low-gravity CFM is a fundamental requirement for establishing a robust space exploration infrastructure, and is applicable across the spectrum of exploration objectives and goals. CFM is the dominant theme of depots for long-term storage and use of highenergy propellants and other fluids. Cryogenic fluid management technologies represent a capability supporting a spiraling and expanding development philosophy towards a robust, reliable, reusable, efficient, and flexible space infrastructure for transportation, operations, commercialization, and science. CFM enables operational flexibility and tolerance to failure, and is particularly suited for spiral investments in evolving capabilities.
The ISCPD systems concept addresses these features in a robust, approach that can enable the synergistic future incorporation of in situ resources as these become available.

\section{Glossary of Acronyms}

CAT Cryogenic Analysis Tool

CER Cost Estimating Relationship

CFM Cryogenic Fluid Management

EELV Evolved Expendable Launch Vehicle

ETO Earth-to-Orbit (Transportation)

FTE Full Time Equivalent

GEO Geostationary Earth Orbit

HLLV Heavy-Lift Launch Vehicle

ISCPD In-Space Cryogenic Propellant Depot

ISS International Space Station

kW kilowatt

kWh kilowatt-hours

LAD Liquid Acquisition Device

LEO Low Earth Orbit

LOX Liquid Oxygen

MEO Middle Earth Orbit

NASA National Aeronautics and Space Administration

PMAD Power Management and Distribution

R\&D Research and Development

SPG Solar Power Generation

TFD Technology Flight Demonstration

TPI Terrestrial Planet Imager

TRL Technology Readiness Level(s)

ZBO Zero Boil Off 


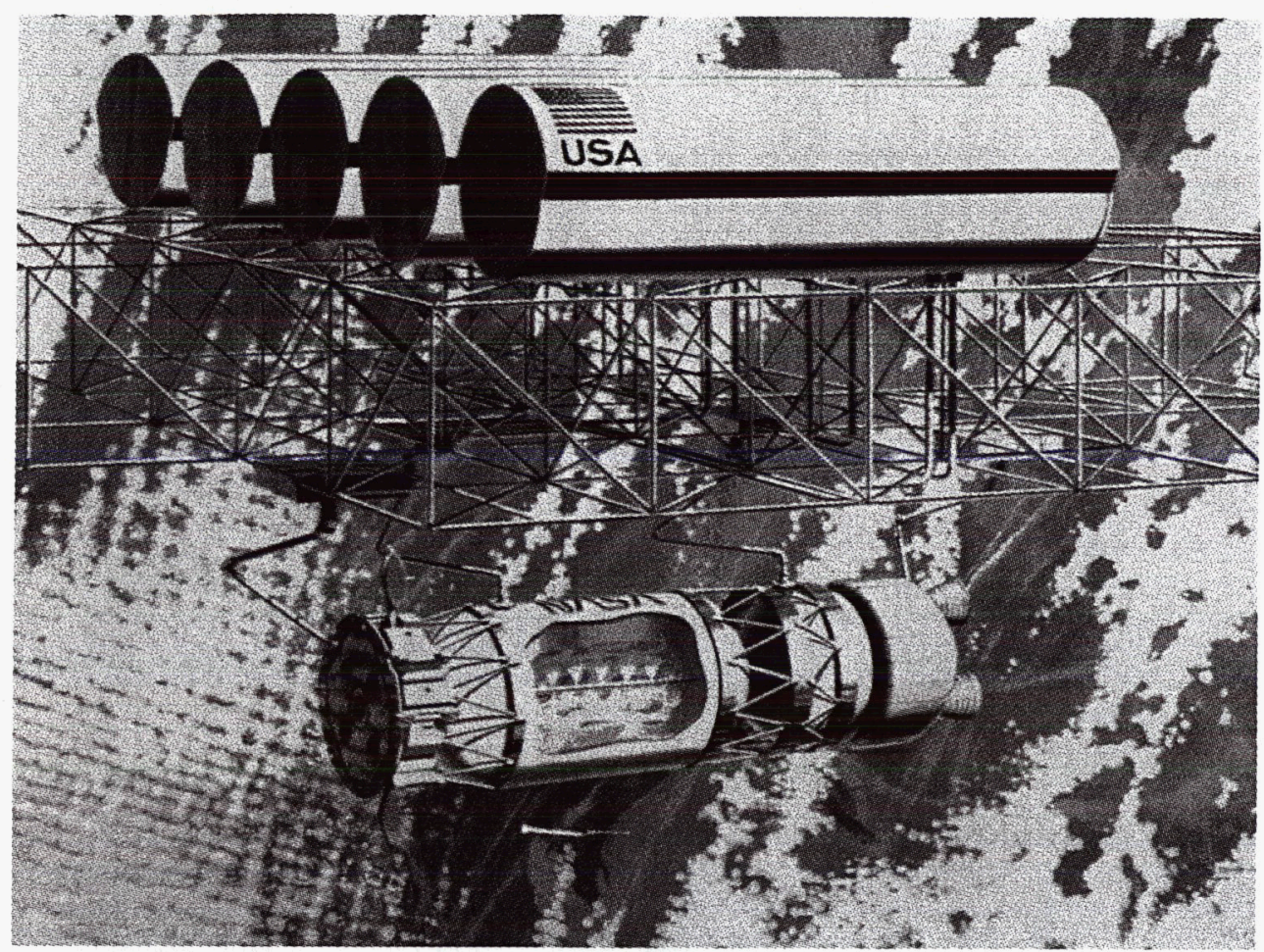

Figure 1 One Concept for a LEO-based In Space Cryogenic Propellant Depot

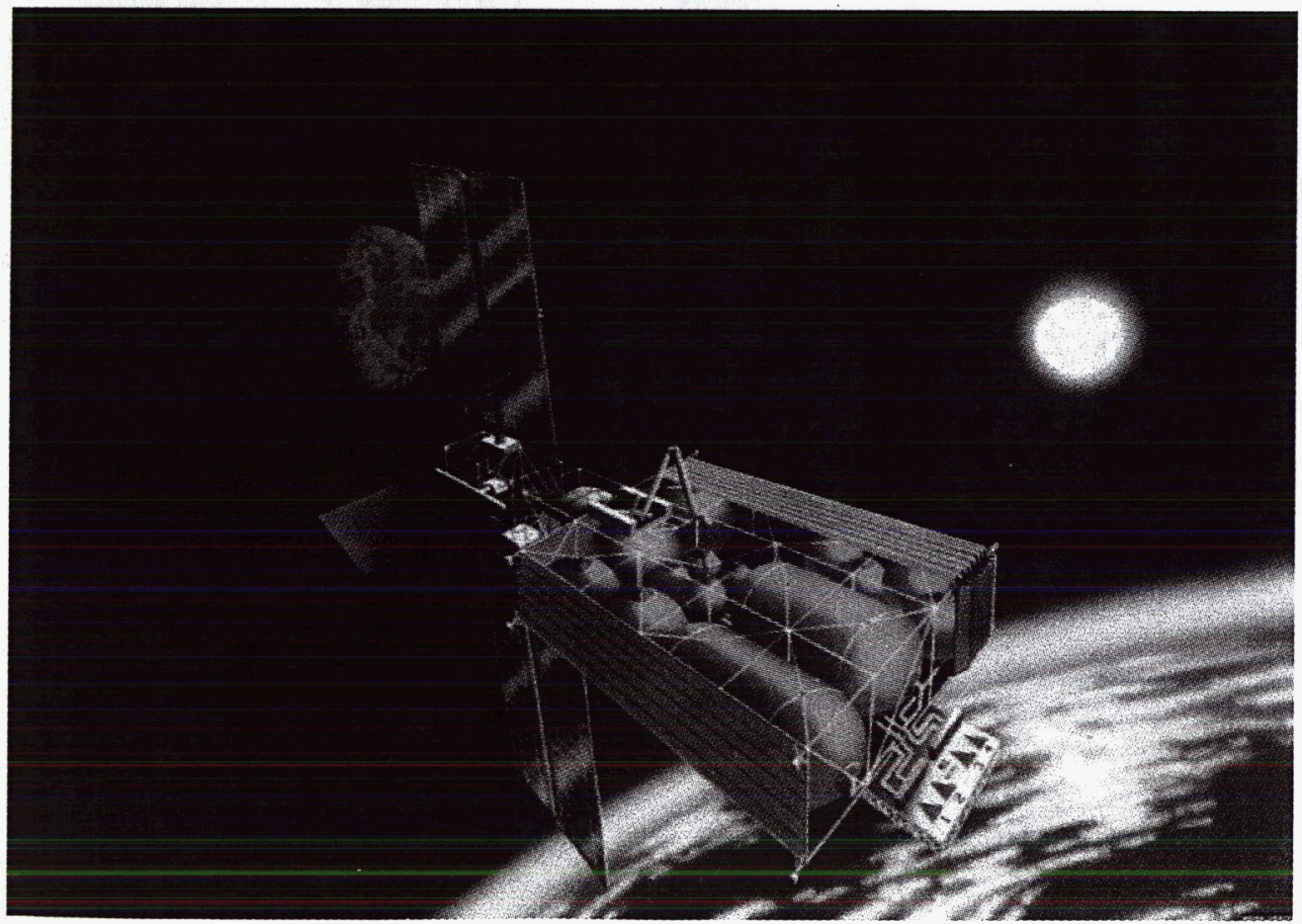

Figure 1 Another Concept for a LEO-based In Space Cryogenic Propellant Depot 


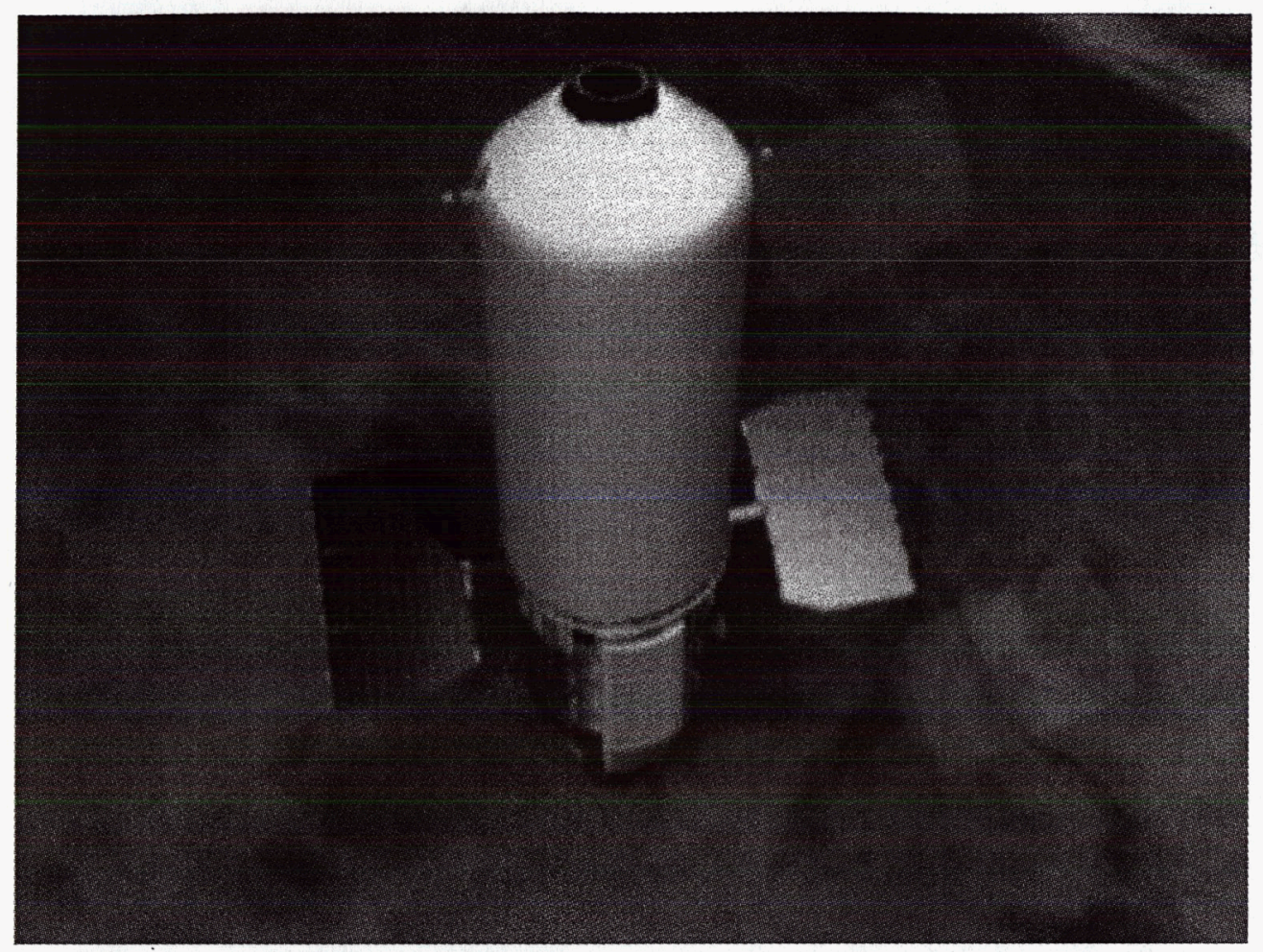

Figure 3 The Hybrid Propellant Module (HPM) - a Modular approach to ISCPD

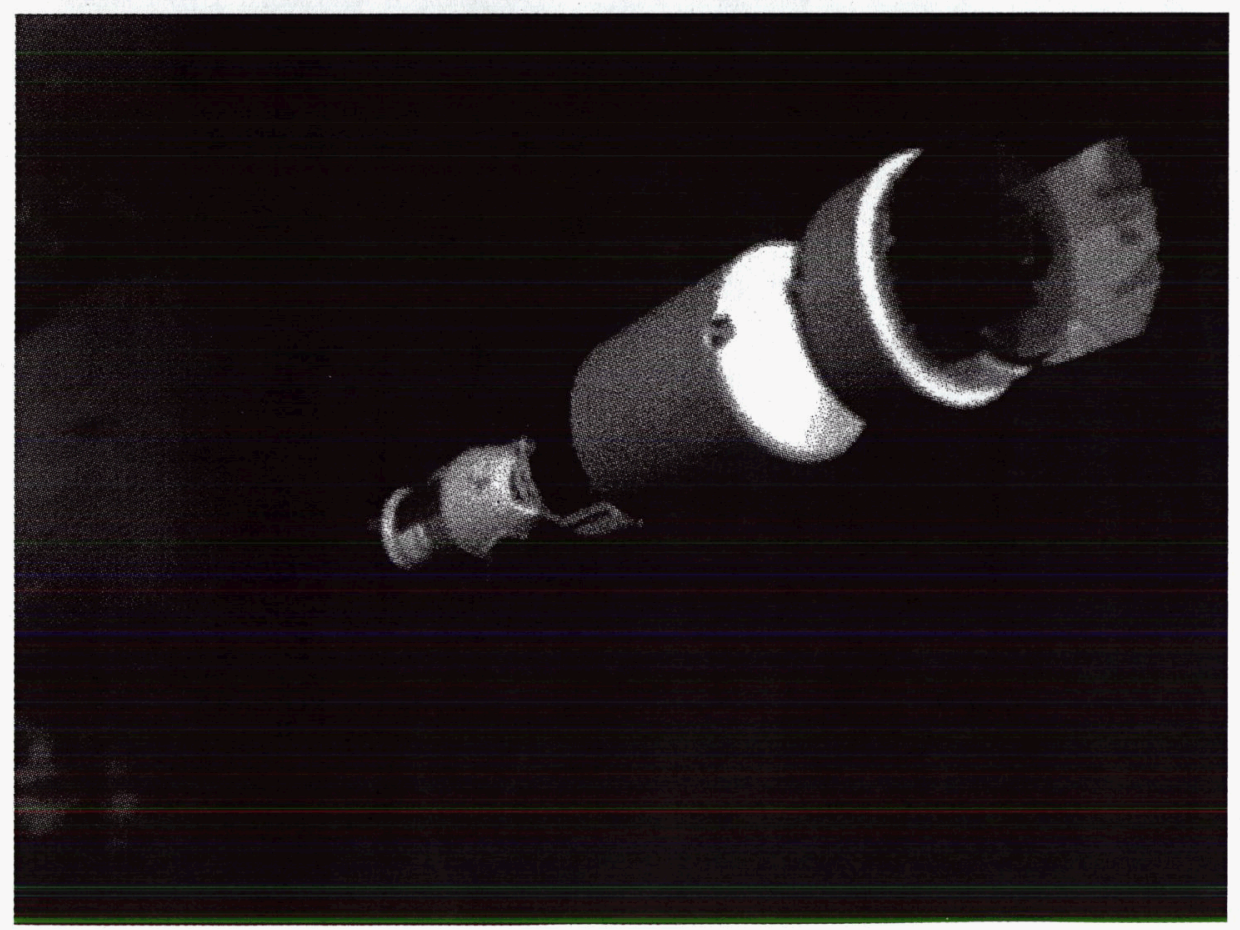

Figure 4 The HPM integrated with other system elements to form an Orbit Transport Vehicle (OTV) 


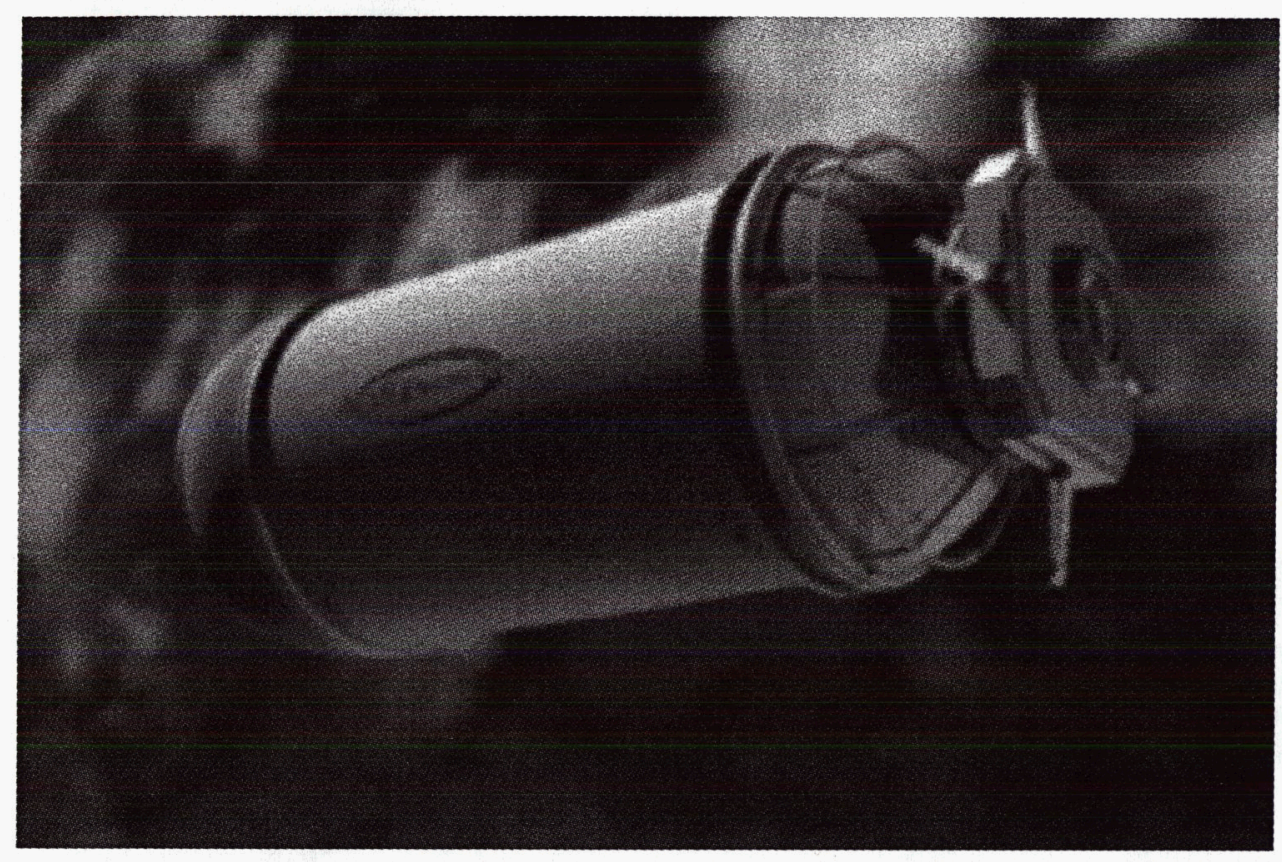

Figure 5 A typical 25-ton class expendable cryogenic propellant tanker (launched in a modular fashion using a commercial EELV) 
$\frac{1}{0}$

들

-

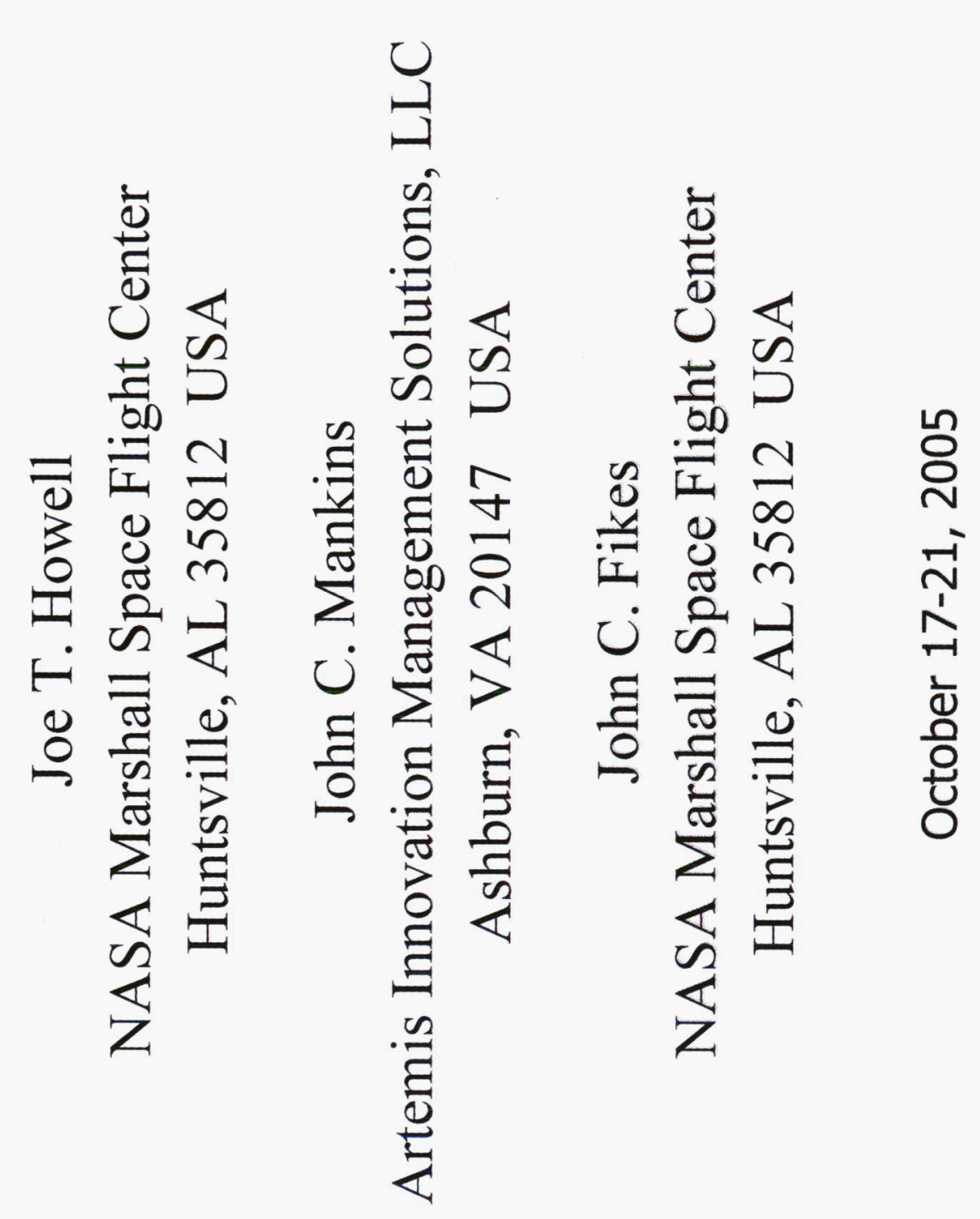

岗 


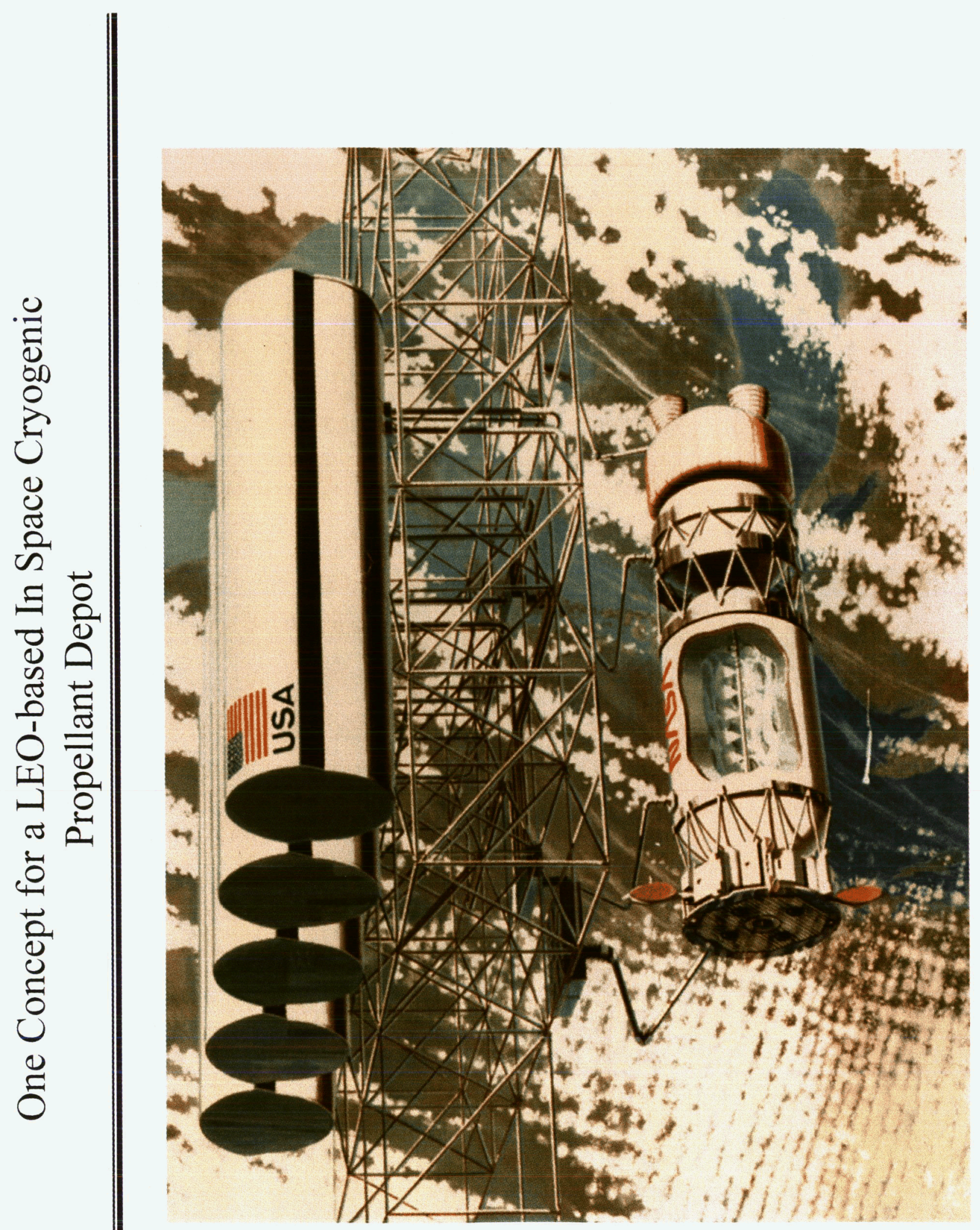




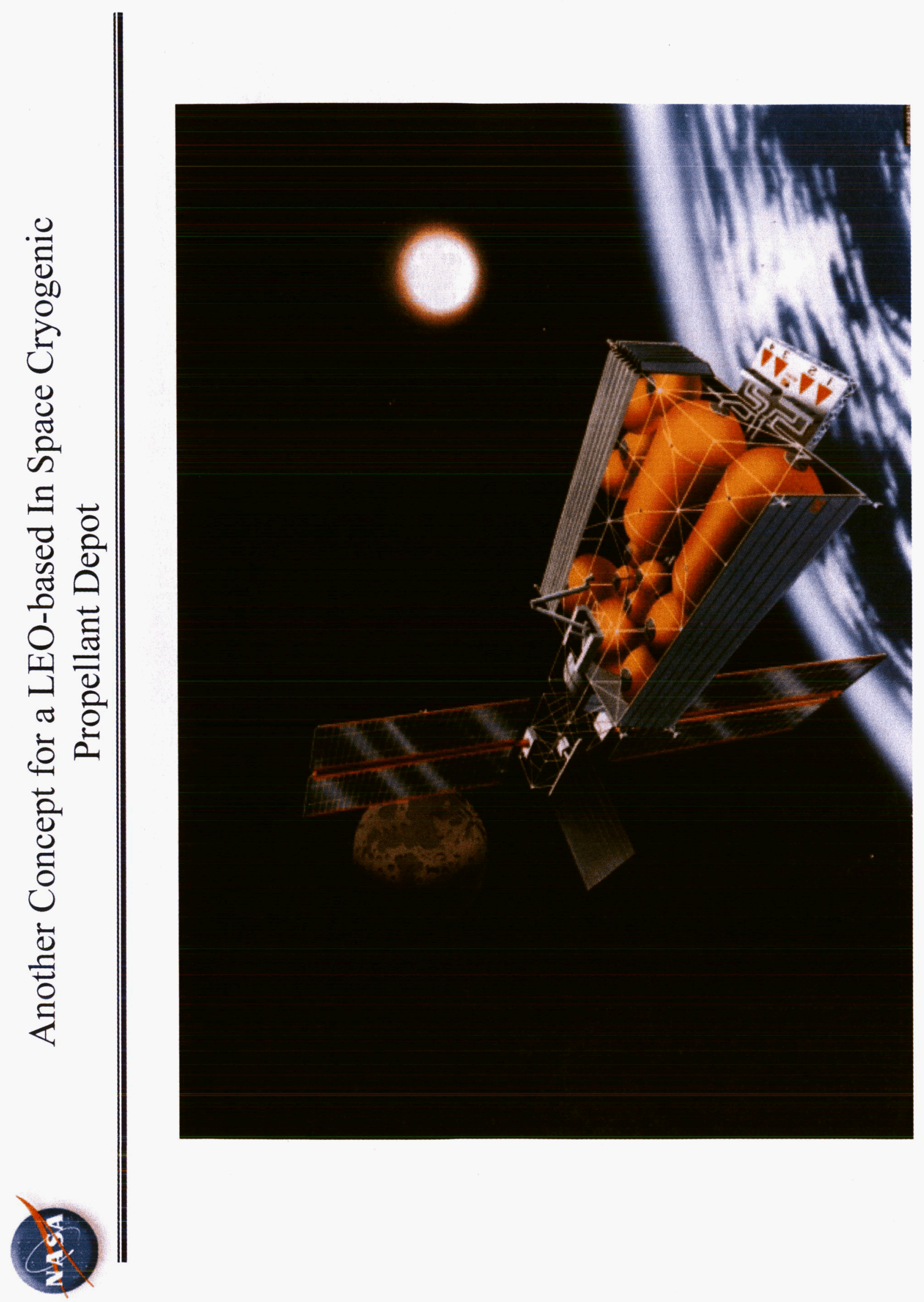




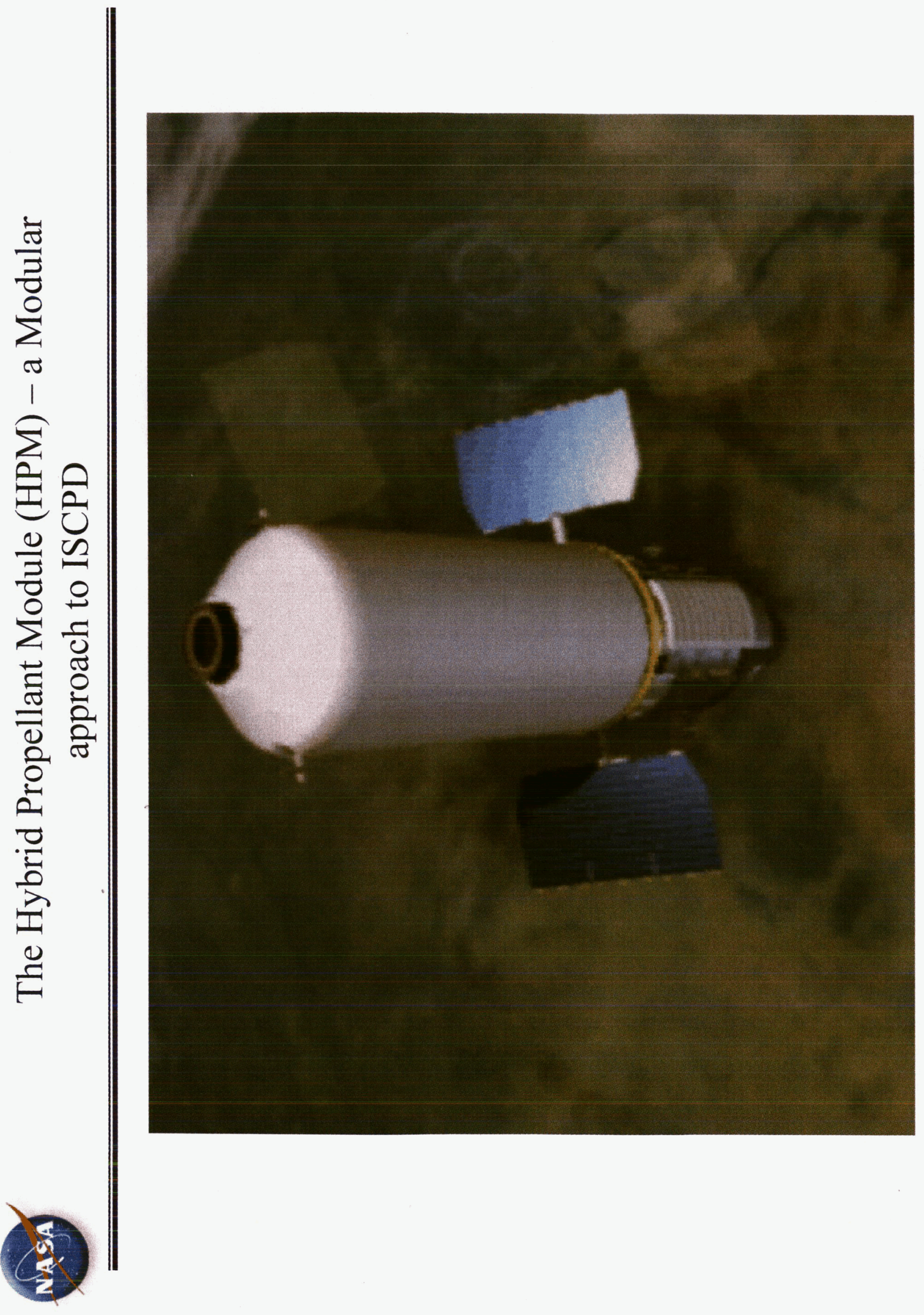




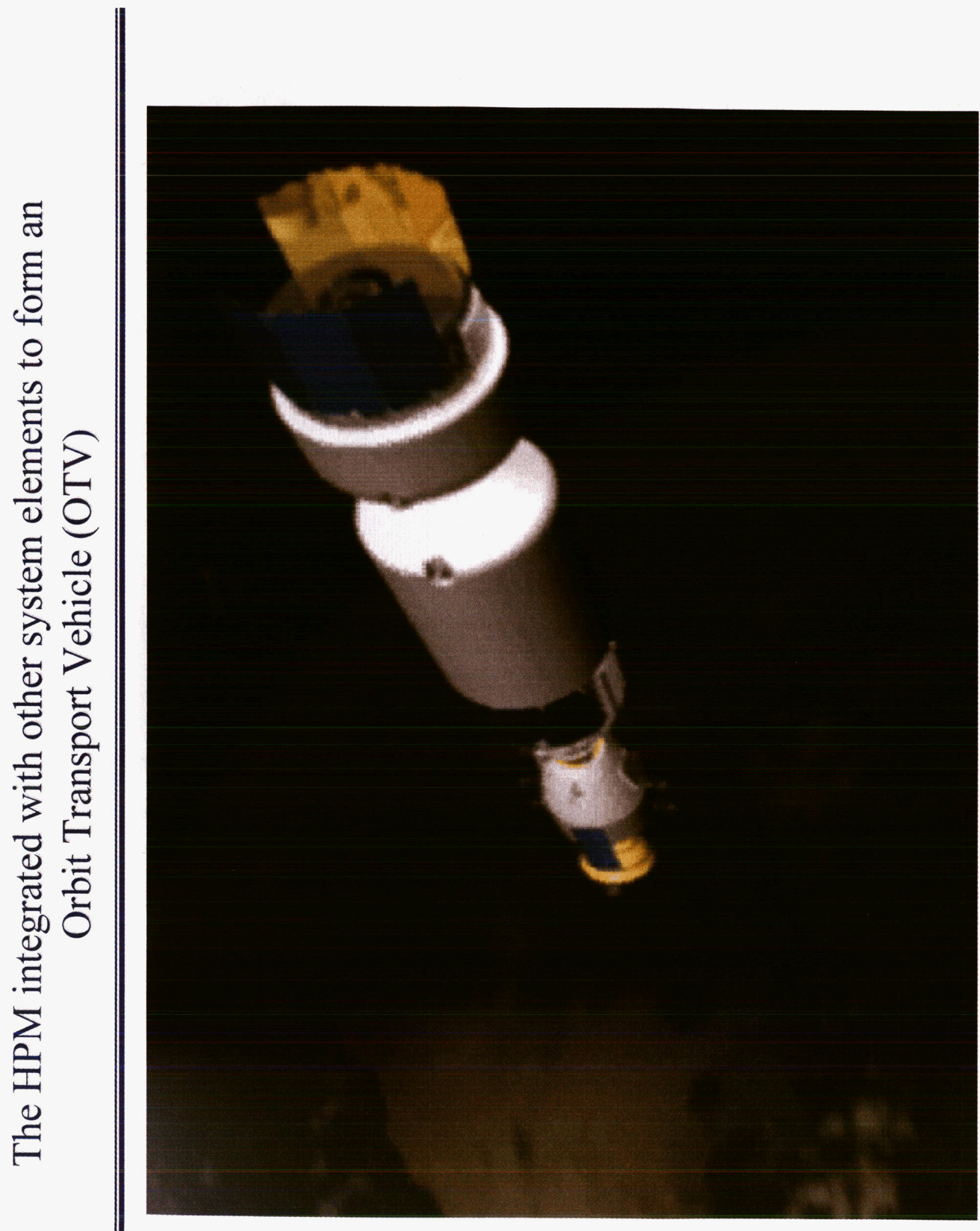




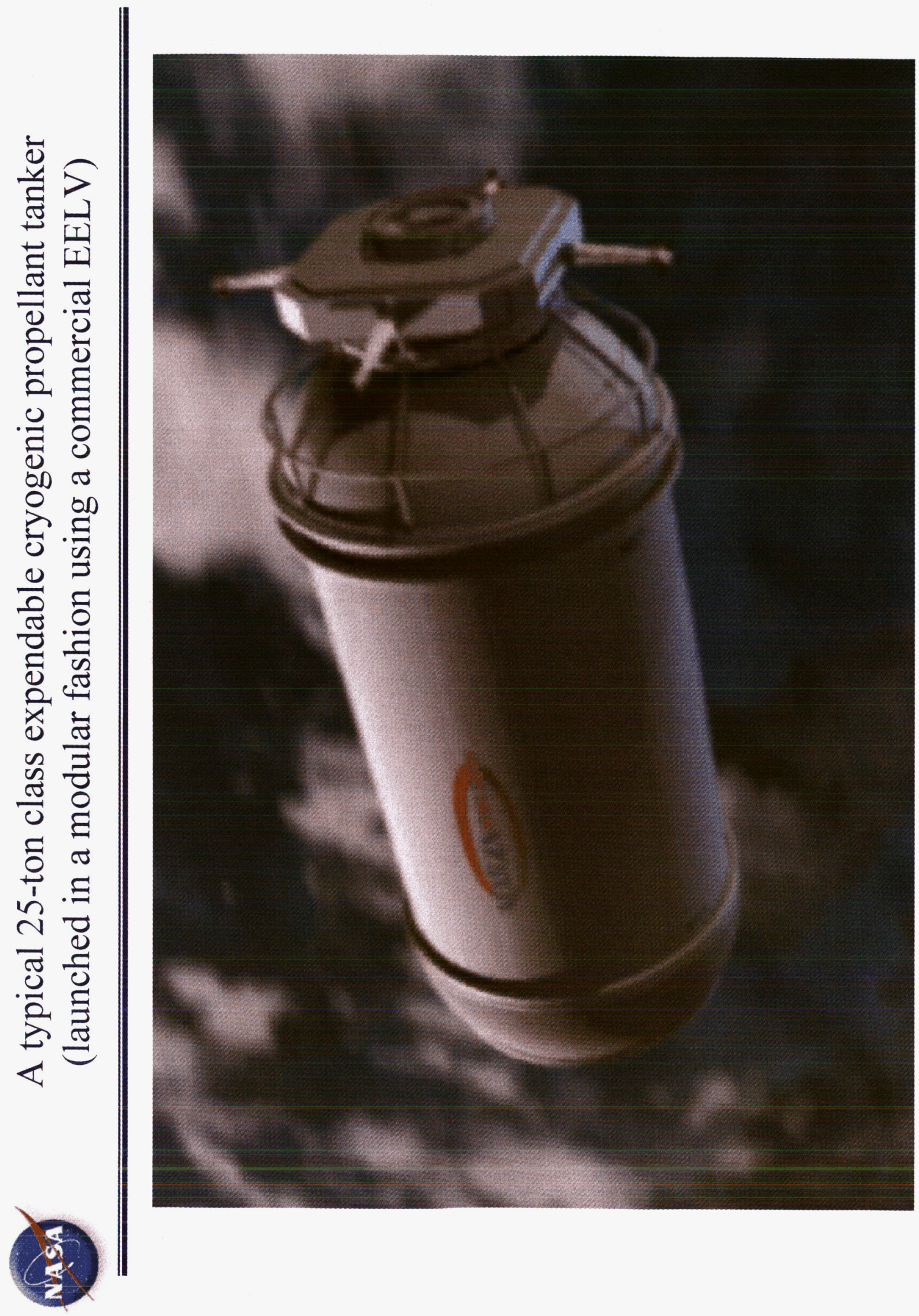

